Résumés des conférences et travaux

\title{
Introduction aux humanités numériques
}

\author{
Peter A. Stokes
}

\section{(2) OpenEdition Journals}

\section{Édition électronique}

URL : https://journals.openedition.org/ashp/3276

DOI : $10.4000 /$ ashp.3276

ISSN : 1969-6310

\section{Éditeur}

Publications de l'École Pratique des Hautes Études

\section{Édition imprimée}

Date de publication : 1 septembre 2019

Pagination : 495-496

ISSN : 0766-0677

\section{Référence électronique}

Peter A. Stokes, «Introduction aux humanités numériques », Annuaire de l'École pratique des hautes études (EPHE), Section des sciences historiques et philologiques [En ligne], 150 | 2019, mis en ligne le 12 juin 2019, consulté le 06 juillet 2021. URL : http://journals.openedition.org/ashp/3276 ; DOI : https:// doi.org/10.4000/ashp.3276 


\title{
INTRODUCTION AUX HUMANITÉS NUMÉRIQUES
}

\author{
Directeur d'études : M. Peter A. Stokes
}

Les humanités numériques (HN) font l'objet de nombreux malentendus : pour quelques-uns, elles se limitent à l'utilisation et au développement de logiciels tels que des tableurs, ou à l'utilisation et / ou la production de sites web ou d'outils informatiques, ou une version « douce » de recherche sans aucun enjeu de recherche mais visant seulement à développer des ressources. En réalité, les HN sont une discipline scientifique, avec ses objectifs propres, ses enjeux et ses méthodologies, une discipline qui est encore en phase de définition et qui trouve différentes déclinaisons dans des contextes différents, mais qui repose sur une base commune de méthodes et d'approches épistémologiques comme la modélisation, la représentation, la pérennisation, la relation. En particulier, les HN s'occupent de la numérisation du patrimoine culturel et des problèmes qui résultent de la « transmédialisation », c'est-à-dire les changements de perception et de compréhension qui dérivent du changement de média et de ce qu'on perd et gagne dans le processus de transformation des objets et des textes complexes. En outre, les HN nous aident à mieux comprendre les objets du monde réel à travers la création de modèles et de représentations numériques qu'on peut manipuler et altérer pour tester des hypothèses et des théories. Les $\mathrm{HN}$ s'occupent aussi de comprendre de quelle façon les objectifs disciplinaires peuvent être redéfinis par l'utilisation de l'ordinateur, en un mot, de voir ce qu'on peut faire d'innovant avec l'ordinateur et avec les données en format numérique dans les différents domaines disciplinaires des sciences humaines. Les $\mathrm{HN}$ ne réduisent donc pas à la capacité de créer des sites web ou des ressources numériques : on les retrouve plutôt dans la création de modèles de données qui sont à la base de ces ressources, et dans l'étude d'une heuristique et d'une ergonomie visant à exploiter ces modèles conceptuels. Par ailleurs, les HN sont aussi « pratiques » : elles « passent» par les doigts et le clavier, pas seulement par les yeux. Cela n'implique pas que les spécialistes dans le domaine des sciences humaines doivent se transformer en informaticiens, bien au contraire, mais pour comprendre effectivement comment les ordinateurs marchent vraiment et envisager les possibilités ouvertes par le moyen numérique, il est fondamental d'avoir une certaine expérience de l'encodage et la programmation. Les HN sont un savoir-faire, ainsi qu'un savoir-penser.

Pour ce cours, nous avons accueilli des étudiants de différentes disciplines et domaines, qu'ils soient en master, ou en doctorat, des chercheurs professionnels, bibliothécaires ou autres. En commençant par des discussions sur la nature des humanités numériques, nous avons abordé des questions relatives aux «livres électroniques » et à la publication électronique : ce que signifie travailler avec des livres numériques plutôt qu'imprimés, comment la recherche est rendue possible et limitée par la numérisation des livres physiques. Ensuite, nous avons considéré les différents formats (HTML, PDF, ePub, et ainsi de suite) et les possibilités et enjeux de chacun, ainsi que les moyens de les créer en pratique. Un autre aspect clé des humanités 
numériques concerne les éditions numériques des textes patrimoniaux. Il s'agit généralement de balisage des textes utilisant la norme internationale XML élaborée par la « Text Encoding Initiative» (TEI), et nous avons fait certains travaux pratiques à cet effet. Cependant, la TEI soulève également d'importantes questions théoriques et méthodologiques : comment avoir un standard qui s'adapte à tous les types d'édition (critique, diplomatique, etc.) ainsi qu'aux différentes théories du texte? Les humanités numériques impliquent souvent de trouver un équilibre entre la flexibilité nécessaire pour les sciences humaines et la régularisation la mieux adaptée à l'ordinateur. En effet, la modélisation est un autre sujet que nous avons abordé : aucune étude ou modélisation d'un objet original ne peut jamais être complète, qu'elle s'exprime en mots imprimés ou dans une représentation numérique formelle ou dans un site web. Le défi consiste donc à déterminer quels aspects de l'objet d'étude sont nécessaires pour répondre aux questions de recherche posées, puis comment ces aspects peuvent être représentés d'une manière qui les rende adaptés à l'étude. Dans un contexte numérique, cela signifie représenter l'objet comme une série de zéros et de uns, et cela implique par conséquent une série de représentations abstraites. Comprendre ces représentations est essentiel pour comprendre les possibilités offertes par les ordinateurs et pour comprendre et utiliser les résultats qu'ils produisent. Nous avons expérimenté les possibilités et les défis de ces principes à travers une série de sujets plus appliqués, tels que la cartographie et les systèmes d'information géographique, la conception et la création de bases de données relationnelles, et les bases du fonctionnement du matériel informatique et de sa programmation. Pour de nombreux chercheurs en sciences humaines, cela est également pertinent pour le traitement automatique des langage (TAL), à savoir l'utilisation de méthodes automatiques pour analyser la langue écrite; bien que ces approches soient largement utilisées et souvent efficaces pour les langues modernes, leurs limites deviennent rapidement évidentes lorsqu'on essaie de les appliquer aux langues historiques et «minoritaires 》 comme celles généralement étudiées à l'École pratique. Les photographies numériques ellesmêmes sont des abstractions et des représentations qui intègrent des hypothèses sur le but des images. Plus précisément, la plupart des appareils photo numériques d'aujourd'hui sont conçus pour que les gens prennent des photos de leurs vacances à la plage plutôt que des images scientifiques de documents. Les implications théoriques et pratiques qui en découlent, comme celles de toutes les autres hypothèses, peuvent être importantes pour la recherche et doivent être comprises par tous. 